\title{
Comparison of contrast medium and atrial pacing as tests of ventricular function in coronary artery disease
}

\author{
BRUCE H. BRUNDAGE AND JAMES E. FARR
}

From the Department of Medicine, Cardiovascular Division, and the Cardiovascular Research Institute, University of California, San Francisco, California 94143; and Fitzsimmons Army Medical Center, Denver, Colorado, and Walter Reed Army Medical Center, Washington, DC, USA

SUMMARY Ventricular function curves were constructed in 39 patients having coronary arteriography by plotting left ventricular end-diastolic pressure and stroke work index before, during, and immediately after atrial pacing. Similar curves were constructed from measurements obtained before and 3 minutes after a standard left ventricular angiogram. Both methods vary preload sufficiently to produce detectable changes in stroke work index, atrial pacing by decreasing the diastolic filling period, and contrast by increasing venous return. The 2 methods were compared for their relative sensitivity in detecting abnormal ventricular function in coronary artery disease. Atrial pacing ventricular function curves were obtained from 8 patients with no heart disease. The left ventricular end-diastolic pressure decreased from 10 to $6 \mathrm{mmHg}$, and stroke work index from 50 to $41 \mathrm{~g} / \mathrm{m}$ per $\mathrm{m}^{2}$. Contrast ventricular function curves had a similar slope, with left ventricular end-diastolic pressure increasing from 8 to $18 \mathrm{mmHg}$ and stroke work index from 50 to $66 \mathrm{~g} / \mathrm{m}$ per $\mathrm{m}^{2}$. In 19 patients with coronary artery disease and normal resting haemodynamics, atrial pacing ventricular function curves did not differ from those of normal subjects. However, contrast ventricular function curves in these same patients were significantly depressed compared with those of normal subjects. The preload stress was similar, but the change in stroke work index was reduced $\left(47\right.$ to $53 \mathrm{~g} / \mathrm{m}$ per $\left.\mathrm{m}^{2}\right)(\mathrm{P}<0.025)$. The remaining 12 patients with coronary artery disease had abnormal ventricular function curves by atrial pacing and contrast, but had recognisable ventricular dysfunction at rest. The preload stress test of contrast medium is more sensitive in detecting abnormal ventricular function in coronary artery disease than atrial pacing.

The assessment of ventricular function in coronary artery disease is important in selecting patients for coronary bypass surgery. The left ventricular angiogram has become a standard part of this evaluation. Ventricular function curves can also be constructed from the response to the angiogram if left ventricular end-diastolic pressure and cardiac output are measured before and several minutes after the procedure. The hyperosmotic contrast medium produces an increase in ventricular preload (Brundage and Cheitlin, 1973, 1974). Atrial pacing also alters preload and has been used to develop similar ventricular function curves. Pacing does not change cardiac output, so increasing heart rate decreases stroke volume and left ventricular end-diastolic pressure (Linhart, 1971; Parker et al., 1971). Since both techniques

Received for publication 2 May 1977 seek to evaluate the Frank-Starling relation, this study was carried out to compare the sensitivity of each method for detecting abnormal ventricular function in coronary artery disease. The response to contrast media appears to be superior to atrial pacing.

Thirty-nine people, 36 men and 3 women, undergoing coronary arteriography to evaluate chest pain, to prepare for, or to assess the results of coronary bypass surgery, comprise the study group. Individuals with rheumatic, congenital, cardiomyopathic, or pericardial disease were excluded. No one received digitalis for 7 days or propranolol for 2 days before the study. The patients were premedicated with $50 \mathrm{mg}$ pethidine and $25 \mathrm{mg}$ promethazine. Lignocaine was used for local anaesthesia.

A balloon-flow-directed thermodilution catheter was introduced into the right femoral vein by 250 
percutaneous technique and positioned in the pulmonary artery. A polyurethane or polyethylene pigtail catheter was placed in the aorta and subsequently the left ventricle via the right femoral artery. A No. 6 bipolar pacing catheter was inserted in the right median antecubital vein and positioned in the right atrium. Pressure was measured through the fluid-filled catheters connected to a Statham p23Db strain gauge on an Electronics for Medicine DR 12 recorder. Left ventricular end-diastolic pressure was recorded at high amplification with a paper speed of $100 \mathrm{~mm} / \mathrm{s}$ and measured at the nadir after the ' $a$ ' wave or at the peak of the $R$ wave of the QRS. It was averaged for 10 beats (Braunwald et al., 1956).

Blood pressure was measured in the ascending aorta. Caraiac outputs were measured in duplicate by the thermodilution technique (Ganz et al., 1971). Duplicate cardiac output measurements that varied more than 10 per cent were repeated.

Atrial pacing was performed with a bipolar pacing catheter in the right atrium, starting at a rate of 15 beats per minute greater than the resting heart rate, and increasing at 2 -minute intervals by 15 beats per minute until the patient experienced Wenckeback atrioventricular blcck or attained a rate of 160 beats per minute. No patient in this study had angina as the end point for pacing.

Cardiac output, left ventricular end-diastolic pressure, and mean aortic pressure were recorded before, at maximum heart rate, and immediately after atrial pacing. After a minimum of 5 minutes, or when left ventricular end-diastolic pressure had returned to control levels, cardiac output, left ventricular enddiastolic pressure, and aortic mean pressure were measured again. Next, a single plane left ventricular angiogram was performed using $0.8 \mathrm{ml} / \mathrm{kg}$ body weight or $60 \mathrm{ml}$ (whichever was the smaller) of methylglucamine diatriazoate (Renografin-76), injected through the pigtail catheter over 4 seconds. The patient was returned to the supine position and cardiac output, left ventricular end-diastolic pressure, and aortic mean pressure were recorded 3 to 5 minutes later. Finally, coronary arteriograms were performed by the Judkins technique. Coronary artery disease was considered significant if one or more major coronary arteries' diameter were narrowed by more than 50 per cent.

Stroke work index (SWI) was calculated by the formula:

SWI $=$

SVI $\times($ mean aortic pressure - LVEDP $) \times 1.36$

100

$\mathrm{g} / \mathrm{m}$ per $\mathrm{m}^{2}$.

Ventricular function curves were constructed by plotting stroke work index against left ventricular end-diastolic pressure. Data for these curves were derived from atrial pacing and the response to contrast medium.

\section{Results}

Eight of the 39 patients, designated group 1, had no evidence of heart disease and their response to atrial pacing and contrast serves as control. All remaining 31 patients had significant coronary artery disease. A normal atrial pacing test was defined as a normal resting left ventricular end-diastolic pressure (12 $\mathrm{mmHg}$ or less), a decrease or no left ventricular enddiastolic pressure increase during pacing, and an overshoot after pacing no greater than $6 \mathrm{mmHg}$ above the baseline. This definition is based on data from other reports on atrial pacing ventricular function curves (Sowton et al., 1967; Linhart et al., 1969; Linhart, 1971; Parker et al., 1971) and this study's controls. Nineteen coronary disease patients (group 2) had a normal atrial pacing test. The remaining 12 patients (group 3) had an abnormal atrial pacing test. The results are listed in the Table. Ventricular function curves for each group were constructed from the 3 points obtained from atrial pacing and from the 2 points derived at the time of angiography (Fig. 1, 2, and 3).

The data were analysed by the paired and unpaired $t$ test and discriminate analysis using the Statistical Package for the Social Sciences (SPSS)Version 650 (Nie et al., 1975).

Fig. 1 depicts the curves obtained in the control patients (group 1). During pacing, left ventricular end-diastolic pressure fell from 9 to $6 \mathrm{mmHg}$, with a corresponding decrease in stroke work index from 49 to $41 \mathrm{~g} / \mathrm{m}$ per $\mathrm{m}^{2}$. Immediately after pacing, stroke work index increased to $50 \mathrm{~g} / \mathrm{m}$ per $\mathrm{m}^{2}$ and left ventricular end-diastolic pressure to $10 \mathrm{mmHg}$. Three to 5 minutes after left ventricular angiography, left ventricular end-diastolic pressure increased from 8 to $18 \mathrm{mmHg}$ and stroke work index from 50 to $66 \mathrm{~g} / \mathrm{m}$ per $\mathrm{m}^{2}$.

Fig. 2 compares the ventricular function curve of patients with normal pacing tests (group 2) with that of the control patients (group 1). Because of the selection process, the atrial pacing ventricular function curves do not differ from normal. However, the ventricular function curve developed from the response to angiography in group 2 is depressed when compared with group 1 . Left ventricular enddiastolic pressure is the same for both groups after angiography (18 $\mathrm{mmHg}$ ), but stroke work index is significantly lower in group 2 (53 vs $66 \mathrm{~g} / \mathrm{m} \mathrm{per}^{2}$, $P<0.025$ ).

Patients in group 3 had an abnormal atrial pacing 
Table Results of haemodynamic response to atrial pacing and contrast medium

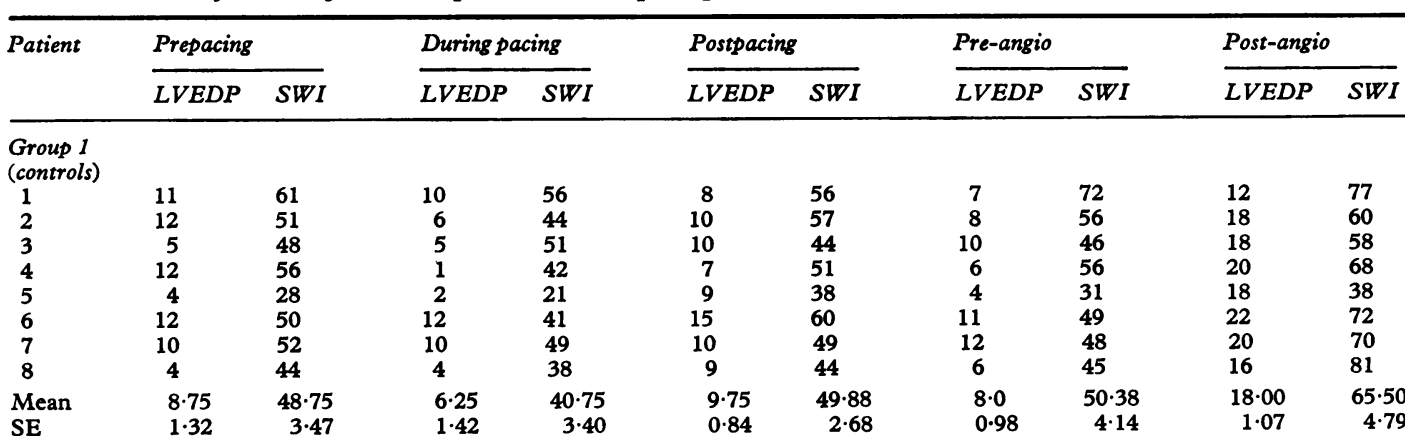

Group 2: coronary artery disease, normal atrial pacing test

\begin{tabular}{|c|c|c|c|c|c|c|c|c|c|c|}
\hline 1 & 5 & 52 & 1 & 35 & 38 & 40 & 9 & 49 & 15 & 48 \\
\hline 2 & 12 & 36 & 10 & 22 & 18 & 31 & 13 & 35 & 20 & 36 \\
\hline 3 & 12 & 62 & 1 & 48 & 10 & 57 & 12 & 72 & 23 & 58 \\
\hline 4 & 12 & 63 & 8 & 38 & 13 & 42 & 10 & 49 & 23 & 39 \\
\hline 5 & 12 & 54 & 8 & 46 & 16 & 58 & 15 & 49 & 20 & 62 \\
\hline 6 & 10 & 49 & 8 & 30 & 10 & 45 & 8 & 54 & 14 & 60 \\
\hline 7 & 9 & 34 & 6 & 25 & 10 & 26 & 10 & 22 & 20 & 25 \\
\hline 8 & 12 & 60 & 3 & 47 & 12 & 54 & 5 & 73 & 20 & 83 \\
\hline 9 & 10 & 45 & 6 & 36 & 10 & 56 & 11 & 47 & 19 & 70 \\
\hline 10 & 7 & 35 & 2 & 30 & 9 & 40 & 6 & 38 & 18 & 43 \\
\hline 11 & 10 & 47 & 7 & 32 & 11 & 43 & 10 & 52 & 13 & 56 \\
\hline 12 & 6 & 26 & 3 & 18 & 8 & 34 & 4 & 30 & 10 & 37 \\
\hline 13 & 12 & 57 & 7 & 38 & 15 & 58 & 12 & 47 & 22 & 56 \\
\hline 14 & 8 & 42 & 2 & 29 & 9 & 49 & 6 & 42 & 18 & 52 \\
\hline 15 & 3 & 34 & 1 & 24 & 10 & 44 & 8 & 38 & 18 & 43 \\
\hline 16 & 12 & 38 & 1 & 40 & 15 & 49 & 13 & 51 & 25 & 54 \\
\hline 17 & 8 & 42 & 2 & 34 & 8 & 51 & 6 & 45 & 12 & 58 \\
\hline 18 & 8 & 45 & 8 & 27 & 12 & 42 & 7 & 42 & 23 & 52 \\
\hline 19 & 10 & 54 & 11 & 49 & 10 & 70 & 10 & 60 & 11 & 76 \\
\hline $\begin{array}{l}\text { Mean } \\
\text { SE }\end{array}$ & $\begin{array}{l}9.37 \\
0.62\end{array}$ & $\begin{array}{r}46.05 \\
2.44\end{array}$ & $\begin{array}{l}5.00 \\
0.78\end{array}$ & $\begin{array}{r}34.11 \\
2.09\end{array}$ & $\begin{array}{r}11 \cdot 26 \\
0.67\end{array}$ & $\begin{array}{r}46.79 \\
2.46\end{array}$ & $\begin{array}{l}9 \cdot 21 \\
0 \cdot 70\end{array}$ & $\begin{array}{r}47 \cdot 11 \\
2 \cdot 88\end{array}$ & $\begin{array}{r}18 \cdot 11 \\
1.02\end{array}$ & $\begin{array}{r}53.05 \\
3.28\end{array}$ \\
\hline \multicolumn{11}{|c|}{ Group 3: coronary artery disease, abnormal pacing test } \\
\hline $\begin{array}{l}1 \\
2\end{array}$ & 13 & 39 & 9 & 32 & 17 & 43 & 18 & 38 & 22 & 49 \\
\hline $\begin{array}{l}2 \\
3\end{array}$ & 13 & 43 & 12 & 28 & 23 & 36 & 20 & 44 & 30 & 46 \\
\hline $\begin{array}{l}3 \\
4\end{array}$ & 13 & 47 & 6 & 50 & 15 & 42 & 13 & 40 & 18 & 56 \\
\hline $\begin{array}{l}4 \\
5\end{array}$ & 22 & 49 & 14 & 35 & 34 & 49 & 14 & 65 & 29 & 60 \\
\hline $\begin{array}{l}5 \\
6\end{array}$ & 15 & 64 & 11 & 40 & 18 & 48 & 14 & 58 & 20 & 69 \\
\hline $\begin{array}{l}6 \\
7\end{array}$ & 15 & 35 & 9 & 24 & 22 & 23 & 20 & 32 & 36 & 41 \\
\hline $\begin{array}{l}7 \\
8\end{array}$ & 19 & 52 & 14 & 52 & 26 & 64 & 21 & 57 & 28 & 74 \\
\hline $\begin{array}{l}8 \\
9\end{array}$ & 6 & 70 & 4 & 54 & 25 & 66 & 11 & 86 & 30 & 77 \\
\hline $\begin{array}{r}9 \\
10\end{array}$ & 8 & 39 & 10 & 32 & 18 & 41 & 13 & 38 & 22 & 38 \\
\hline $\begin{array}{l}10 \\
11\end{array}$ & 6 & 57 & 12 & 56 & 19 & 54 & 6 & 76 & 25 & 70 \\
\hline $\begin{array}{l}11 \\
12\end{array}$ & 26 & 41 & 35 & 37 & 29 & 52 & 10 & 61 & 35 & 38 \\
\hline 12 & 20 & 27 & 12 & 31 & 19 & 30 & 12 & 33 & 27 & 27 \\
\hline $\begin{array}{l}\text { Mean } \\
\text { SE }\end{array}$ & $\begin{array}{r}15.25 \\
1.86\end{array}$ & $\begin{array}{r}46.92 \\
3.56\end{array}$ & $\begin{array}{r}12.93 \\
2.23\end{array}$ & $\begin{array}{r}39 \cdot 25 \\
3 \cdot 18\end{array}$ & $\begin{array}{r}22.08 \\
1.61\end{array}$ & $\begin{array}{r}46 \cdot 50 \\
3 \cdot 27\end{array}$ & $\begin{array}{r}14 \cdot 17 \\
1.29\end{array}$ & $\begin{array}{r}52.33 \\
5.08\end{array}$ & $\begin{array}{r}26.83 \\
1.63\end{array}$ & $\begin{array}{r}53 \cdot 75 \\
4 \cdot 73\end{array}$ \\
\hline
\end{tabular}

LVEDP, left ventricular end-diastolic pressure; SWI, stroke work index.

test (Fig. 3). These patients, compared with the normals (group 1), had an abnormal resting left ventricular end-diastolic pressure (15 vs $9 \mathrm{mmHg}$, $\mathrm{P}<0.02)$, which decreased with pacing $(12 \mathrm{mmHg}$ ). Post-pacing left ventricular end-diastolic pressure increased an average of $7 \mathrm{mmHg}$ above baseline in this group. This overshoot was the most distinct of any group (Table). There was a decrease in stroke work index with pacing ( 47 to $40 \mathrm{~g} / \mathrm{m}$ per $\mathrm{m}^{2}$ ), but post-pacing stroke work index was the same as before pacing. The resultant pacing ventricular function curve is shifted to the right of normal and is flattened, indicating significant depression of ventricular function in this group. The ventricular $\stackrel{\mathcal{D}}{f}$ function curve from the haemodynamic response to contrast was likewise abnormal.

Because we had to reuse some measurements to compare the 3 patient groups, multiple $t$ tests can $\frac{\vec{D}}{\mathrm{D}}$ produce erroneously optimistic significant differences (Zar, 1974). Therefore, stepwise discriminate 


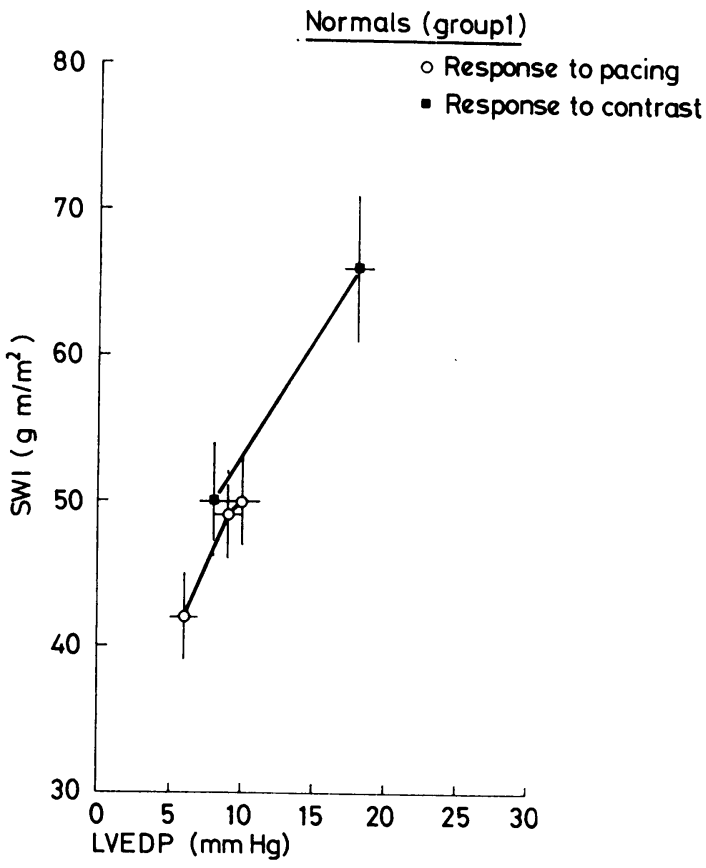

Fig. 1 Comparison of contrast medium and atrial pacing ventricular function curves in patients with no evidence of heart disease (group 1).

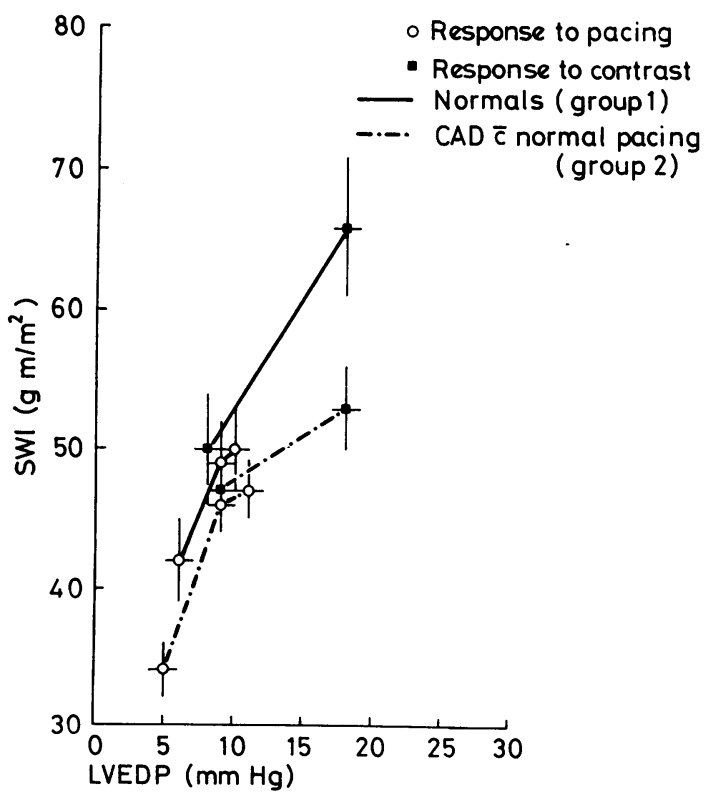

Fig. 2 Comparison of contrast medium and atrial pacing ventricular function curves in group 1 and group 2 patients.

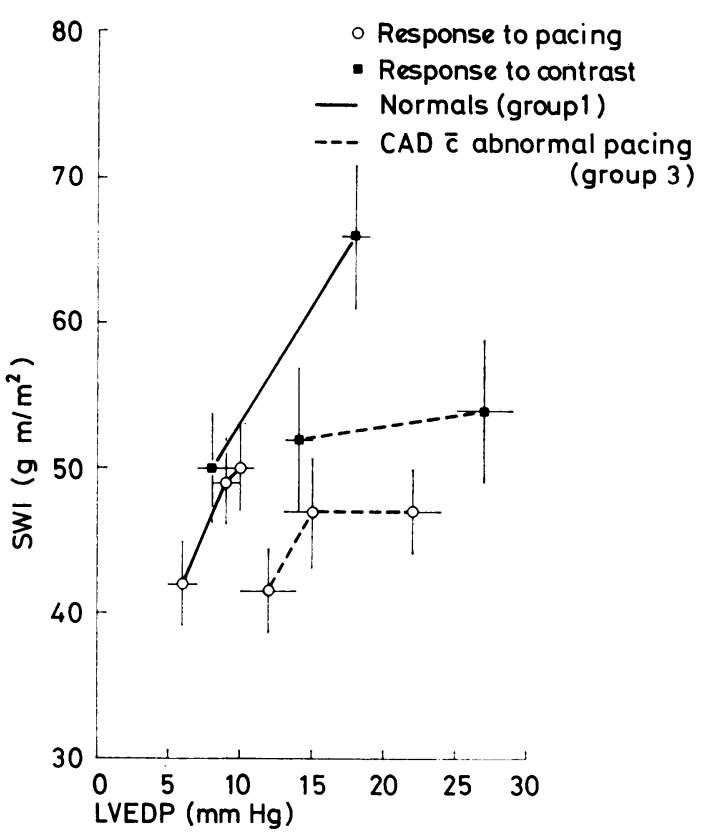

Fig. 3 Comparison of contrast medium and atrial pacing ventricular function curves in group 1 and group 3 patients.

analysis was applied to see which technique, atrial pacing or the response to contrast medium, was most powerful in discriminating between normal and coronary artery disease patients. Atrial pacing data alone did not distinguish normal from coronary artery disease patients $\left(\chi^{2}=10 \cdot 01, P=0 \cdot 188\right)$. However, data from the response to contrast could significantly $\left(\chi^{2}=11.40, P=0.044\right)$ separate normal and coronary artery disease patients. Also, stroke work index after angiography was the single most potent discriminator (Wilks' $\lambda=0.900, P=0.050$, change in Rao's V $=4 \cdot 10, \mathrm{P}=0.043$ ) between the two groups.

\section{Discussion}

Ventricular function curves derived from the cardiac response to atrial pacing have been reported by others (Linhart, 1971; Parker et al., 1971). Because cardiac output remains constant during atrial pacing, stroke volume decreases with increase in rate. This is accompanied by a decrease in left ventricular end-diastolic pressure. After the abrupt termination of pacing, stroke volume and left ventricular end-diastolic pressures return to baseline values, or there is a small overshoot. These changes make it possible to construct a ventricular function curve. More recently, function curves have 
been constructed from the haemodynamic response to contrast media used during ventriculography (Brundage and Cheitlin, 1974). This is possible because 3 to 5 minutes after left ventriculography, left ventricular end-diastolic pressure and stroke volume increase, with little change in blood pressure (Brundage and Cheitlin, 1974). These changes are the result of increased plasma osmolarity (Giammona et al., 1963), and blood volume (Iseri et al., 1965) caused by the hyperosmotic contrast medium. These events produce increased venous return, which increases ventricular end-diastolic dimension and, by the Frank-Starling mechanism, stroke volume is increased (Brundage, 1976; Tyberg et al., 1976; Hamby et al., 1977).

Since both techniques depict part of the FrankStarling curve, we investigated the relative sensitivity of the 2 tests in detecting abnormal ventricular function which accompanied coronary artery disease. Fig. 1 shows that in normal patients (group 1), before each intervention, the stroke work index and left ventricular end-diastolic pressure were essentially the same. Atrial pacing produced movement down and up one ventricular function curve. The haemodynamic response to contrast produced movement up the curve. The slopes of the 2 curves are similar, indicating that each intervention characterises a different part of the same curve.

Atrial pacing has been said to depict a steeper than normal ventricular function curve because of the Bowditch phenomenon (Linhart et al., 1969; Parker et al., 1969). However, Noble et al. (1969) and Higgins et al. (1973) found little or no positive inotropic effect from pacing at physiological heart rates. Our results are in agreement with the latter findings.

In addition, it is reported that ventricular function curves produced from the response to contrast media are depressed, compared with those produced by other osmotic loads, suggesting a negative inotropic effect on the myocardium (Cohn et al., 1973). However, others have failed to show any depressant effect of contrast medium on myocardial contractility lasting more than 1 minute after injection, and the measurements in our study were performed 3 to 5 minutes after angiography (Tyberg et al., 1976). Moreover, the data from group 1 suggest that atrial pacing and the response to contrast are depicting different portions of the same function curve in normals.

Fig. 2 compares the 19 coronary disease patients with normal atrial pacing tests (group 2) with the normals (group 1). The values for left ventricular end-diastolic pressure and stroke work index at each phase of the pacing procedure do not differ significantly, so the pacing ventricular function curves are similar. However, in group 2 the function curve produced from the response to contrast media is distinctly depressed, compared with group 1 . Though the change in left ventricular end-diastolic pressure is almost the same, increase in stroke work index is less in group 2 (53 vs $66 \mathrm{~g} / \mathrm{m}$ per $\mathrm{m}^{2}$, $P<0.025)$. These results indicate that the response to contrast media in these coronary disease patients is more sensitive than atrial pacing in detecting abnormal ventricular function. The discriminate analysis we performed confirms this observation.

The third group were coronary disease patients with abnormal atrial pacing tests (Fig. 3). Collectively, stroke work index and left ventricular enddiastolic pressure decreased with pacing, as in the other 2 groups. However, because resting left ventricular end-diastolic pressure was raised, the pacing curve was shifted to the right. After pacing, the overshoot of left ventricular end-diastolic pressure was most pronounced in this group, though stroke work index was unchanged. These results produced a flattened ventricular function curve. Similarly, the curve constructed from the response to contrast was depressed and shifted to the right (Fig. 3).

Both interventions show depressed ventricular function curves in group 3, but such tests may not be necessary to detect abnormal function, since resting haemodynamics, such as left ventricular enddiastolic pressure, are often abnormal. It is patients of the type described in group 2 who often require some form of stress test, in order to detect abnormal ventricular function. It is here that this study indicates that the function curves derived from the response to contrast media are more sensitive than pacing curves. Our results are similar to those of Khaja et al. (1970), who found exercise function curves superior to pacing curves in coronary disease patients.

Atrial pacing is most successful in detecting abnormal ventricular function in coronary disease if angina is induced by the tachycardia. None of our patients developed angina during pacing, though many had angina sufficient to require coronary bypass surgery. The explanation for this finding is not apparent, as adequate heart rates were achieved in our patients. However, Rios and Hurwitz (1974) also found atrial pacing to be a poor stress for producing symptomatic ischaemia.

It is expected that the angina state will produce measurable deterioration of ventricular function. However, there is a need for a means of evaluating ventricular function in coronary artery disease in the absence of symptomatic ischaemia. Does coronary bypass surgery improve ventricular function? This controversy is, in part, the result of the differences in methods used to measure ventricular function. If the 
measurements are performed during acute symptomatic ischaemia preoperatively, and are compared with postoperative data obtained in the absence of ischaemia, it is not surprising that the conclusion is reached that surgery has improved ventricular function. It may be just as important to assess preoperative ventricular function in the absence of angina for comparison with postoperative function, particularly if it is to be determined if the operation can improve ventricular function by relieving a state of chronic asymptomatic ischaemia. Techniques, such as measuring the response to contrast medium, may be useful in clarifying this problem (Brundage, et al., 1977).

The portion of the ventricular function curve defined by each intervention may explain, in part, why the response to contrast medium is better than atrial pacing in detecting abnormal function in coronary disease. Atrial pacing defines a lower segment of the function curve than contrast medium and differences may be more readily recognised in the upper portion of function curves, particularly if coronary artery disease decreases left ventricular compliance. The ventricular pressure-volume relation is curvilinear and diminished compliance would be most apparent at larger ventricular volumes. The haemodynamic effect of contrast media is to increase ventricular volume, whereas atrial pacing decreases volume. Therefore the response to contrast would be the more sensitive to changes in compliance.

While an altered ventricular pressure-volume relation may, in part, explain the different sensitivities of contrast and atrial pacing, some data indicate that myocardial contractility is also impaired in coronary patients with depressed function curves (Brundage, 1976).

Whatever the mechanism, the haemodynamic response to contrast is superior to atrial pacing in detecting altered ventricular function in coronary disease.

\section{References}

Braunwald, E., Fishman, A. P., and Cournand, A. (1956). Time relationship of dynamic events in the cardiac chambers, pulmonary artery and aorta in man. Circulation Research, 4, 100-107.

Brundage, B. H. (1976). End diastolic stretch in coronary artery disease (abstract). Circulation, 54, Suppl. II, 66.

Brundage, B. H., Anderson, W. T., Dovia, J. E., Cheitlin, M. D., and de Castro, C. M. (1977). Determinants of ventricular function following coronary bypass surgery. American Heart fournal, 93, 687-698.

Brundage, B. H., and Cheitlin, M. D. (1973). Left ventricular angiography as a function test. Chest, 64, 70-74.

Brundage, B. H., and Cheitlin, M. D. (1974). Ventricular function curves from the cardiac response to angiographic contrast. American Heart fournal, 88, 281-288.

Cohn, P. F., Horn, H. R., Teicholz, L. E., Kreulen, T. H.,
Herman, M. V., and Gorlin, R. (1973). Effects of angiographic contrast medium on left ventricular function in coronary artery disease. American fournal of Cardiology, 32, 21-26.

Ganz, W., Donoso, R., Marcus, H. S., Forrester, J. S., and Swan, H. J. C. (1971). A new technique for measurement of cardiac output by thermodilution in man. American fournal of Cardiology, 27, 392-396.

Giammona, S. T., Lurie, P. R., and Segar, W. E. (1963). Hypertonicity following selective angiocardiography. Circulation, 28, 1096-1101.

Hamby, R. I., Aintablian, A., Wisoff, G. B., and Hartstein, M. L. (1977). Effects of contrast medium on left ventricular pressure and volume with emphasis on coronary artery disease. American Heart fournal, 93, 9-18.

Higgins, C. B., Vatner, S. F., Franklin, D., and Braunwald, E. (1973). Extent of regulation of the heart's contractile state in the conscious dog by alteration in the frequency of contractions. Fournal of Clinical Investigation, 52, 1187-1194.

Iseri, L. T., Kaplan, M. A., Evans, M. J., and Nickel, E. D. (1965). Effects of concentrated contrast media during angiography on plasma volumes and plasma cs nolality. American Heart Fournal, 69, 154-158.

Khaja, F., Parker, J. O., Ledwich, R. J., West, R. O., and Armstrong, P. W. (1970). Assessment of ventricular function on coronary artery disease by means of atrial pacing and exercise. American fournal of Cardiology, 26, 107-116.

Linhart, J. W., Hildner, F. J., Barold, S. S., and Samet, P. (1969). Myocardial function in patients with coronary artery disease. American fournal of Cardiology, 23, 379-386.

Linhart, J. W. (1971). Pacing-induced changes in stroke volume in the evaluation of myocardial function. Circulation, 43, 253-261.

Nie, N. H., Hull, C. H., Jenkins, J. G., Steinbrunner, K., and Bent, D. H. (1975). Discriminate analysis. In Statistical Package for the Social Sciences, p. 434. Ed. by K. J. Bowman and $M$. Cahill. McGraw-Hill, New York.

Noble, N. I. M., Wyler, J., Milne, E. N. C., Trenchard, D., and Guz, A. (1969). Effect of changes in heart rate on left ventricular performance in conscious dogs. Circulation Research, 24, 285-295.

Parker, J. O., Khaja, F., and Case, R. B. (1971). Analysis of left ventricular function by atrial pacing. Circulation, 43, 241-252.

Parker, J. O., Ledwich, J., West, R. O., and Case, R. B. (1969). Reversible cardiac failure during angina pectoris: haemodynamic effects of atrial pacing in coronary artery disease. Circulation, 39, 745-757.

Rios, J. C., and Hurwitz, L. E. (1974). Electrocardiographic responses to atrial pacing and multistage treadmill exercise testing: correlation with coronary arteriography. American Fournal of Cardiology, 34, 661-666.

Sowton, G. E., Balcon, R., Cross, D., and Frick, M. H. (1967). Measurement of the anginal threshold using atrial pacing; a new technique for the study of angina pectoris. Cardiovascular Research, 1, 301-307.

Tyberg, J. V., Mathey, D. G., Stowe, D. F., Chatterjee, K., and Parmley, W. W. (1976). Hemodynamic effects of contrast medium : resultant of phasic changes in loading and contractility (abstract). Circulation, 53 and 54, Suppl. II, 155.

Zar, J. H. (1974). Biostatistical Analysis, p. 130. Prentice-Hall, Englewood Cliffs, New Jersey.

Requests for reprints to Dr Bruce H. Brundage, 1186-Moffitt, University of California, San Francisco, California 94143, USA. 